\title{
Self-Rated Smell Ability Enables Highly Specific Predictors of COVID-19 Status: A Case-Control Study in Israel
}

\author{
Noam Karni, ${ }^{1,5, a}$ Hadar Klein, ${ }^{2, a, \bullet}$ Kim Asseo, ${ }^{2}$ Yuval Benjamini, ${ }^{3}$ Sarah Israel ${ }^{1,5}$ Musa Nammary, ${ }^{1,5}$ Keren Olshtain-Pops, ${ }^{4,5}$ Ran Nir-Paz, ${ }^{4,5, \odot}$ Alon Hershko, ${ }^{1,5}$
} Mordechai Muszkat, ${ }^{1,5, b}$ and Masha Y. Niv ${ }^{2, b, \odot}$

${ }^{1}$ Department of Medicine, Hadassah University Hospital, Mt. Scopus Campus, Jerusalem, Israel, ${ }^{2}$ The Institute of Biochemistry, Food and Nutrition, The Faculty of Agriculture, Food and Environment, The Hebrew University, Rehovot, Israel, ${ }^{3}$ Department of Statistics, The Hebrew University, Mt. Scopus Campus, Jerusalem, Israel, ${ }^{4}$ Department of Clinical Microbiology and Infectious Diseases, Hadassah-Hebrew University Medical Center, Jerusalem, Israel, and ${ }^{5}$ Faculty of Medicine, The Hebrew University of Jerusalem, Jerusalem, Israel

Background. Clinical diagnosis of coronavirus disease 2019 (COVID-19) is essential to the detection and prevention of COVID-19. Sudden onset of loss of taste and smell is a hallmark of COVID-19, and optimal ways for including these symptoms in the screening of patients and distinguishing COVID-19 from other acute viral diseases should be established.

Methods. We performed a case-control study of patients who were polymerase chain reaction-tested for COVID-19 (112 positive and 112 negative participants), recruited during the first wave (March 2020-May 2020) of the COVID-19 pandemic in Israel. Patients reported their symptoms and medical history by phone and rated their olfactory and gustatory abilities before and during their illness on a 1-10 scale.

Results. Changes in smell and taste occurred in 68\% (95\% CI, 60\%-76\%) and 72\% (95\% CI, 64\%-80\%) of positive patients, with odds ratios of 24 (range, 11-53) and 12 (range, 6-23), respectively. The ability to smell was decreased by $0.5 \pm 1.5$ in negatives and by $4.5 \pm 3.6$ in positives. A penalized logistic regression classifier based on 5 symptoms had $66 \%$ sensitivity, $97 \%$ specificity, and an area under the receiver operating characteristics curve (AUC) of 0.83 on a holdout set. A classifier based on degree of smell change was almost as good, with $66 \%$ sensitivity, $97 \%$ specificity, and 0.81 AUC. The predictive positive value of this classifier was 0.68 , and the negative predictive value was 0.97 .

Conclusions. Self-reported quantitative olfactory changes, either alone or combined with other symptoms, provide a specific tool for clinical diagnosis of COVID-19. A simple calculator for prioritizing COVID-19 laboratory testing is presented here.

\section{Graphical Abstract}
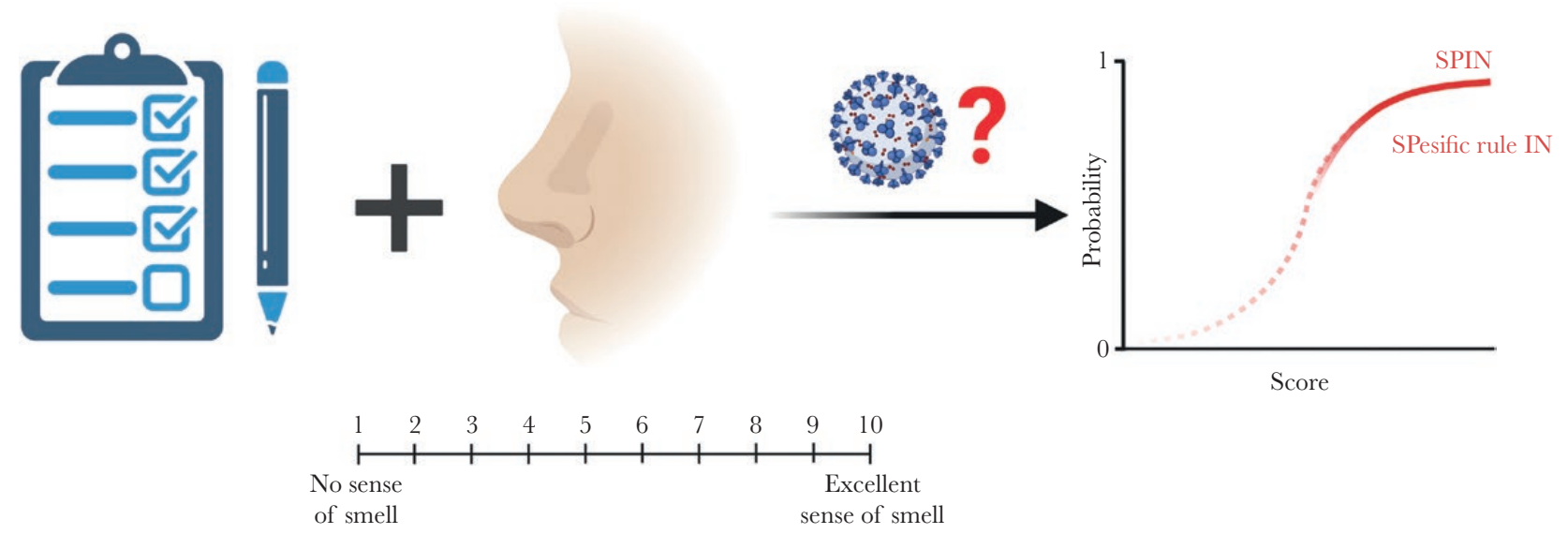
Received 18 August 2020; editorial decision 30 November 2020; accepted 2 December 2020.
'Equal contribution
'Equal contribution
Correspondence: M. Y. Niv, PhD, The Institute of Biochemistry, Food and Nutrition, The
Faculty of Agriculture, Food and Environment, The Hebrew University, Herzl street, Rehovot
7610001, Israel (masha.niv@mail.huji.ac.il).

Open Forum Infectious Diseases ${ }^{\circledR} 2021$

(C) The Author(s) 2020. Published by Oxford University Press on behalf of Infectious Diseases Society of America. This is an Open Access article distributed under the terms of the Creative Commons Attribution-NonCommercial-NoDerivs licence (http://creativecommons.org/licenses/ by-nc-nd/4.0/), which permits non-commercial reproduction and distribution of the work, in any medium, provided the original work is not altered or transformed in any way, and that the work is properly cited. For commercial re-use, please contact journals.permissions@oup.com DOI: 10.1093/ofid/ofaa589 
Keywords. COVID-19; classifier; prediction; smell loss; taste loss.

In December 2019, the first cases of severe acute respiratory syndrome coronavirus 2 (SARS-CoV-2) were reported in Wuhan, China [1]. The resulting coronavirus disease 2019 (COVID-19) has become a global pandemic, with 16.5 million reported cases as of July 29, 2020, according to World Health Organization.

Since March 2020, an increasing number of reports regarding loss of taste and smell in COVID-19 infection have appeared in preprints $[2,3]$ and in the general press, and it is currently well established that loss of taste and smell is common in COVID-19 patients [4-7].

In a recent crowd-sourced study, $\sim 7000$ app users reported testing positive for COVID-19, with $65 \%$ of those reporting that they lost their sense of smell or taste [8], a 3-fold increase in prevalence compared with COVID-19 negatives [9]. The severity of loss of smell and taste in COVID-19 patients is striking: These sensory abilities were reduced by a mean \pm SD of $-79.7 \pm 28.7$ and $-69.0 \pm 32.6$, respectively, as reported by about 4000 participants using a $0-100$ visual analog scale (VAS) [6]. A follow-up study suggested that recent loss of smell is the best predictor for COVID-19 [10].

Here we assess the prevalence of different COVID-19 symptoms as well as the degree and additional characteristics of smell and taste changes in polymerase chain reaction (PCR) swabtested COVID-19-positive vs COVID-19-negative patients. Importantly, patients were recruited in a manner that did not disclose the underlying chemosensory questions in this study. We used these data to develop a classifier that can prioritize patients for PCR testing, help epidemiological investigations, and screen large populations.

\section{METHODS}

\section{Aim and Setting}

This prospective study compared symptoms in real-time (RT) PCR-tested COVID-19-positive and COVID-19-negative patients. Patients with PCR test results (positive or negative) were recruited via social media (Twitter and Facebook) and word of mouth from March 2020 to May 2020 and interviewed from April 2020 to June 2020. The cohort comprised 224 Israeli patients aged $\geq 18$ years (Figure 1 ). The participants were not aware that the questionnaire would include loss of smell and taste before agreeing to partake in the study. Informed consent was obtained from all participants. The study was approved by the Hadassah Medical Center Helsinki Committee (permit number 0236-20-HMO).

\section{General Design}

The interviews were carried out over the phone. The questionnaire is based on questions compiled by physicians and scientists in the Global Consortium for Chemosensory Research (GCCR) [6]. The full questionnaire is included in the Supplementary Data and has 5 parts: (1) General information (eg, age, gender); (2) Medical history (eg, medical conditions, medications, changes in taste/smell in the past, pregnancy, contact with a confirmed patient); (3) Current illness: 23 physical signs and symptoms, including binary questions (yes/no) on smell, taste, and chemesthesis (cooling, burning, tingling sensation), PCR swab test results and dates, date of exposure to confirmed COVID-19 patient, subjective recovery feeling; (4) Smell: participants were instructed to rate their sense of smell/ taste and the degree of their nose blockage on a scale from 1 to 10 ( 1 corresponding to "no sense of smell" and 10 to "excellent sense of smell") and similarly rate the ability to breathe through the nose before/during/after illness. Blocked nose rating was used to test the plausible hypothesis that it causes a change of smell; (5) Taste (eg, rating of taste ability before/during/after illness, as described for smell), experience of strange/bad taste in the mouth, change in sensitivity to irritants (chemesthesis), and change in basic taste modalities - sweet, salty, sour, bitter, each elicited by nonvolatile compounds via specific receptors or channels expressed in dedicated taste receptor cells [11]. The fifth basic taste modality, umami or savory, was not used because it does not have a Hebrew translation. "Other" taste was available as an additional optional answer. Data were kept in Compusense Cloud online software (Compusense Inc., Guelph, ON, Canada).

\section{Statistical Analysis}

Log-odds for the individual symptoms were calculated over the full data set. Confidence intervals and $P$ values for the log-odds were estimated using the $\mathrm{glm}$ function from R (https://www.rproject.org/).

Classifiers were trained from the reported symptoms to evaluate the separation between COVID-19-positive and COVID19-negative patients. The classifiers were trained on a random subset of two-thirds of the data (the training set, 148 samples) and evaluated on the remaining samples (the test set). Sampling of the train and test sets was stratified by COVID-19 status. We trained the classifier on the full symptom matrix: All symptoms of question 23 in the questionnaire (Supplementary Data) were included, except "no symptoms" or "other." All eye symptoms were combined into "eye symptoms." Also added were quantitative questions for taste, smell, and nose blockage (rating before the illness minus rating during the illness, questions 31 , $35,37,38,40$, and 41 in the questionnaire) and chemesthesis (question 45); "coated tongue," "dizziness," "ears pressure," "eye burn," "eye discharge," "hearing change," "lacrimation," and "vision changes" were removed as these symptoms were reported 
by $<10 \%$ of the subjects. The classifiers were trained as penalized logistic regressions using the elastic net algorithm ( $\alpha=.5$ implemented in the glmnet package in the $\mathrm{R}$ environment). This regression method encourages sparse coefficient vectors, meaning that it is suitable in situations where few coefficients are nonzero. The regularization parameter (lambda) was initially set using cross-validation, but then increased until the model included no more than 6 symptoms. For classifiers based on a single symptom, no regularization was used.

Classifiers were evaluated using the holdout test set. The score from the classifier was thresholded at 0 , so that patients with a score exceeding 0 were called positive by the classifier. Sensitivity (predicting COVID-19 positives correctly) and specificity (predicting COVID-19 negatives correctly) metrics were calculated from the following formulas:

$$
\text { Sensitivity }=\frac{T P}{T P+F N}, \quad \text { Specificity }=\frac{T N}{T N+F P},
$$

in which $\mathrm{TP}$ (TN) are the COVID-19 positives (negatives) classified correctly and FN (FP) are the COVID-19 positives (negatives) classified incorrectly. Due to our balanced sample, accuracy is the average of sensitivity and specificity. We further computed the accuracy metrics that account for the expected proportion of positive cases in the tested population, namely the positive predictive value (PPV) and negative predictive value $(\mathrm{NPV})$. The scores obtained from the logistic classifier $(s)$ were translated into probability of being positive $(P)$ by adjusting for the proportion of COVID+ from the tests $(\pi)$ according to the following formulas:

$$
\begin{gathered}
o=\frac{P(+\mid \text { Symptoms })}{P(-\mid \text { Symptoms })}=\exp (s) * \frac{\pi}{1-\pi} ; \\
P=\text { Probability to be positive }=\frac{o}{o+1} .
\end{gathered}
$$

We take $\pi$ to be .08 because that was the proportion during data collection.

The ROC curve corresponds to true-positive and falsepositive rates for different values of the threshold; the curve and the area under the ROC curve (AUC), which measures the degree of separability between positive and negative scores, were estimated using the $p R O C$ package [12].

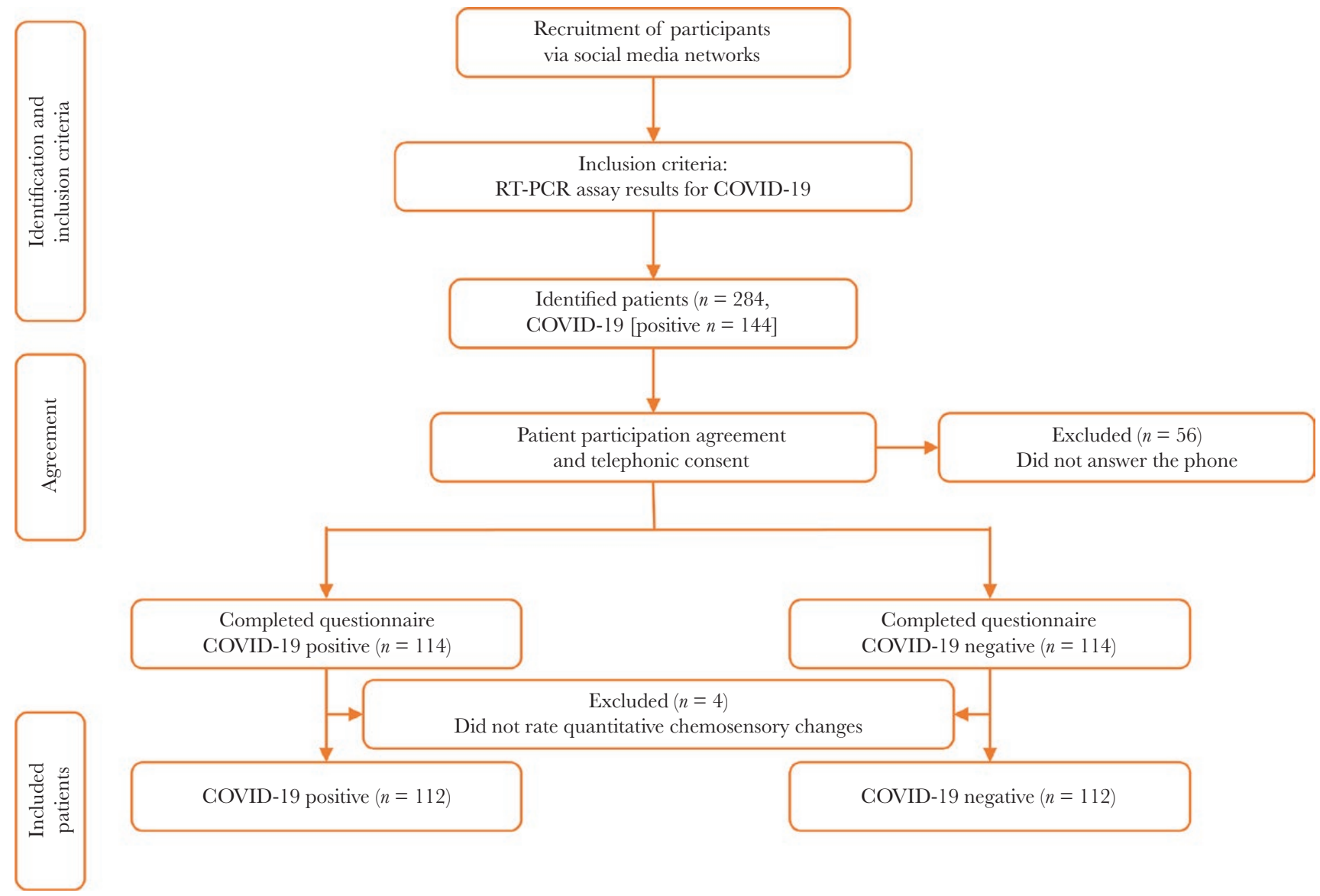

Figure 1. Flowchart of patient selection for the case-control study. Abbreviations: COVID-19, coronavirus disease 2019; RT-PCR, reverse transcription polymerase chain reaction. 


\section{Sample Size Calculation}

The sample size was calculated to allow for detecting differences in smell loss or taste loss prevalence between COVID-19positive and -negative populations. Based on previous research (eg, [13]), we used conservative estimates of $60 \%$ prevalence in the positive population and $35 \%$ prevalence in the negative population. Power was estimated by Monte Carlo simulations, repeatedly $(b=1000)$ resampling from 2 binomial distributions corresponding to the positive population and the negative population. Assuming 100 individuals are assigned to each group and a 2 -sided $t$ test is used, the probability of detection (power) is $92 \%$. To be on the conservative side, we used somewhat larger samples.

\section{RESULTS}

\section{Patients' Characteristics}

Completed questionnaires were obtained from 112 COVID19 positives and 112 COVID-19 negatives. The age of the respondents was $35 \pm 12$ years for positives, and $37 \pm 12$ years for negatives (mean $\pm \mathrm{SD}$ ). The positives group included more men (64\%), while the negatives group was more balanced (48\% males). Seven patients were classified as hospitalized (received respiratory support during their hospitalization and/or were hospitalized in the intensive care unit), and the other 217 were classified as ambulatory patients.

\section{Patients' Symptoms}

Signs and symptoms that appeared in the binary part of the questionnaire (Supplementary Data, question 23 of the full questionnaire) and were found to occur in at least $10 \%$ of the positive patients are summarized in Table 1. A few symptoms, including dry cough and sore throat, were prevalent in COVID19 positives, but these were even more prevalent in the negative control sample.

Smell change, taste change, change in chemesthetic ability (perceiving spicy, tingling, or cooling sensations), and muscle ache were significantly more prevalent in COVID-19-positive as compared with COVID-19-negative patients (68\%, 72\%, $31 \%, 62 \%$ vs $8.0 \%, 18 \%, 6 \%, 34 \%$, respectively) (Table 1 ). Other Centers for Disease Control and Prevention (CDC)-recognized symptoms [14], such as lack of appetite, fever, and diarrhea were $\sim 2$ or 3 times more common among positives than negatives.

Nausea and vomiting, although considered a COVID-19 symptom, were not more common among COVID-19-positive as compared with COVID-19-negative patients. By contrast, lack of appetite, despite not being included as an "official" CDC symptom [14], was found to be significantly more common in COVID-19-positive patients.

Taste and smell changes often, but not always, occured together: Figure 2A shows the distribution of reports on taste and smell changes. Change in both smell and taste perception was reported by $63 \%$ of positive patients and only $6 \%$ of negative patients. Four percent of positive patients experienced only smell change, and $9 \%$ reported taste change with no smell change.

Sixty-three percent of the positive patients reported impairment of at least 1 of the 4 taste modalities (sweet, salty, sour, and bitter) compared with only $10 \%$ among the negative patients (Figure 2B). Six percent of positives and $2 \%$ of negatives added comments about taste changes as free text. An additional $31 \%$ of positives and $88 \%$ of negatives did not report any taste-related changes. In COVID-19-positive patients with taste impairment, all 4 taste modalities were usually impaired.

\section{Chemosensory Change Ratings and Details}

In addition to the binary questions, the participants were asked to rate their senses of smell and taste before and during their illness on a 1-10 scale.

As seen in Figure 3, the change in smell and taste ability during disease, compared with a self-reported individual baseline before the disease, differed greatly between positive and negative patients. Both taste and smell changes were significantly greater for COVID-19-positive patients compared with COVID-19-negative patients (mean \pm SD, $4.5 \pm 3.6$ and $4.9 \pm 3.8$ vs $0.5 \pm 1.5$ and $0.4 \pm 1.5 ; P<.0001$ and $P<.0001$, respectively). When considering only patients who reported taste or smell changes (who answered "yes" to the respective binary questions), the averages in positive patients were $6.3 \pm 2.6$ for taste and $7.1 \pm 2.4$ for smell, compared with negative patients with $2.1 \pm 2.3$ for taste and $4.8 \pm 2.6$ for smell (among patients with any change in taste $P<.0001$, in smell $P=.03$ ).

Combinations of symptoms were next checked for ability to differentiate COVID-19-positive from -negative diagnoses. To that end, several classifiers were trained based on $66 \%$ of the sample and evaluated on the $34 \%$ that was kept as a holdout set. The process of selection of descriptors is outlined in Figure 4A. Relevant symptoms $(n=30)$ were included as possible descriptors for the classifiers, and the elastic-net penalization was increased until no more than 6 symptoms were included in the model (number limited for practicality). The effect of excluding or including a particular symptom was evaluated in order to understand the importance of separating taste from smell and using binary vs quantitative measures for each (Figure 4A).

The results of the evaluation on the holdout set are summarized in Supplementary Table 1, and Classifiers 1-3 can be seen in Figure 4B. Classifiers that did not use chemosensory symptoms had poor performance (AUC, 0.60; black curve, Classifier 1, and additional classifiers) (Supplementary Table 1). Adding the quantitative smell change symptom (maroon curve, Classifier 2) was sufficient to outperform all other classifiers (AUC, 0.83). Remarkably, using quantitative smell change as a sole symptom (magenta curve, Classifier 3 ) resulted in a classifier that was nearly equally effective as Classifier 2 (AUC, 0.81). 


\begin{tabular}{|c|c|c|c|c|}
\hline & Positives, \% & Negatives, \% & PValue & Estimated OR (Lower Bound, Upper Bound) \\
\hline Smell change* & 68 & 8 & $<.0001$ & $24.16(10.98,53.14)$ \\
\hline Taste change* & 72 & 18 & $<.0001$ & $12.02(6.36,22.72)$ \\
\hline Chemesthesis ${ }^{*, a}$ & 31 & 6 & $<.0001$ & $6.82(2.88,16.16)$ \\
\hline Muscle ache & 62 & 34 & $<.0001$ & $3.25(1.88,5.61)$ \\
\hline Lack of appetite & 53 & 27 & $<.0001$ & $3.04(1.74,5.32)$ \\
\hline Fever & 65 & 41 & $<.0001$ & $2.69(1.56,4.61)$ \\
\hline Diarrhea & 24 & 12 & .01 & $2.42(1.17,4.98)$ \\
\hline Abdominal pain & 21 & 11 & .03 & $2.27(1.07,4.81)$ \\
\hline Nausea or vomiting & 21 & 12 & .07 & $1.97(0.94,4.12)$ \\
\hline Eye symptoms & 21 & 12 & .1 & $1.81(0.88,3.73)$ \\
\hline Headache & 62 & 49 & .06 & $1.66(0.98,2.83)$ \\
\hline Productive cough & 46 & 40 & .4 & $1.24(0.73,2.12)$ \\
\hline Bad taste in mouth & 17 & 14 & .6 & $1.23(0.59,2.53)$ \\
\hline Runny nose & 38 & 39 & .9 & $0.96(0.56,1.65)$ \\
\hline Fatigue & 18 & 21 & .6 & $0.84(0.43,1.64)$ \\
\hline Chest pain & 23 & 28 & .5 & $0.79(0.43,1.44)$ \\
\hline Voice change & 13 & 18 & .4 & $0.71(0.34,1.47)$ \\
\hline Dry cough & 62 & 71 & .2 & $0.67(0.38,1.17)$ \\
\hline Sore throat & 37 & 56 & $<.0001$ & $0.45(0.26,0.77)$ \\
\hline Breath difficulty & 21 & 38 & $<.0001$ & $0.43(0.24,0.78)$ \\
\hline
\end{tabular}

Only signs and symptoms that were present in at least $10 \%$ of all study participants are listed. Those with the highest odds ratios are marked with asterisks, and those reported by at least a third of positives are shown in boldface.

a Burning, cooling, or tingling sensation.

Adding taste change to Classifier 2 did not improve its performance, as it resulted in an AUC of 0.82 (Classifier 7) (Supplementary Table 1). Taste change as a sole descriptor resulted in an AUC of 0.75 (Classifier 15) (Supplementary Table 1) and, as an added descriptor to other "basic" symptoms, in an AUC of 0.76 (Classifier 13) (Supplementary Table 1).
Thus, while there is a high correlation (0.82) between quantitative changes in smell and quantitative changes in taste, the smell change descriptor outperforms the taste descriptor. Using the quantitative smell and taste descriptors resulted in higher AUCs than binary (yes/no) descriptors of these changes. For example, a binary smell descriptor used as a sole descriptor resulted in an
A

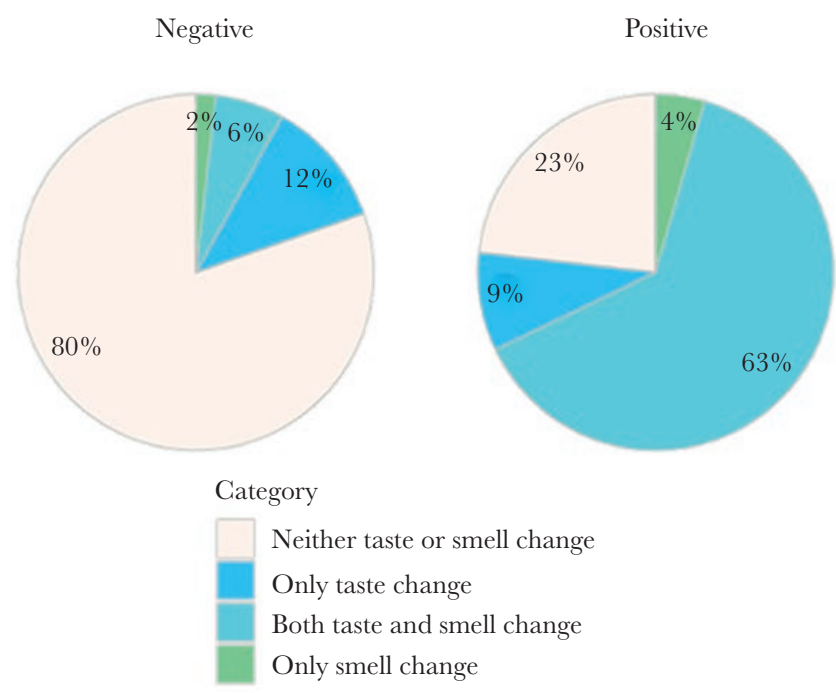

B

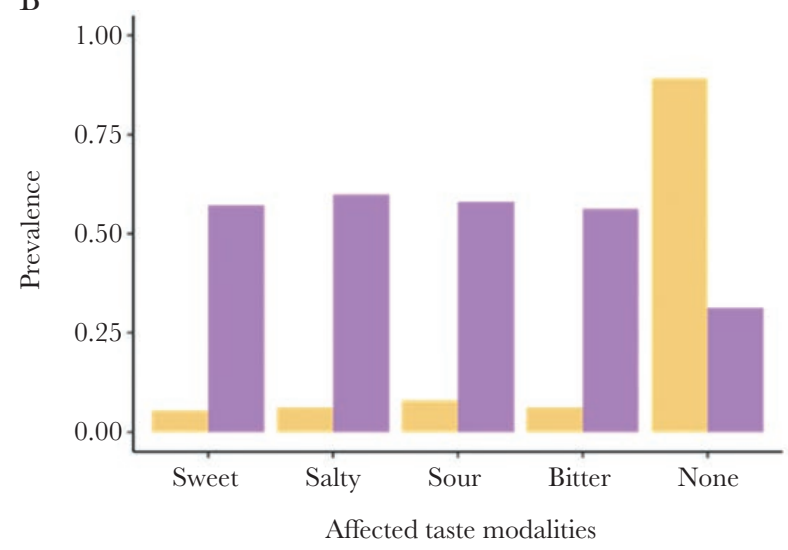

Diagnosis Negative Positive

Figure 2. Smell, taste, and taste modality changes during disease. A, The pie chart presents the prevalence of smell and taste changes in positive and negative patients, occurring together or separately. Numbers indicate the percentage of COVID-19 positives and negatives reporting taste or smell loss (blue and green, respectively), both taste and smell loss (turquoise), or neither (seashell). B, The prevalence of the 4 taste modalities in COVID-19-positive and -negative patients. COVID-19 positives are represented in purple, and COVID-19 negatives are represented in orange. Abbreviation: COVID-19, coronavirus disease 2019. 


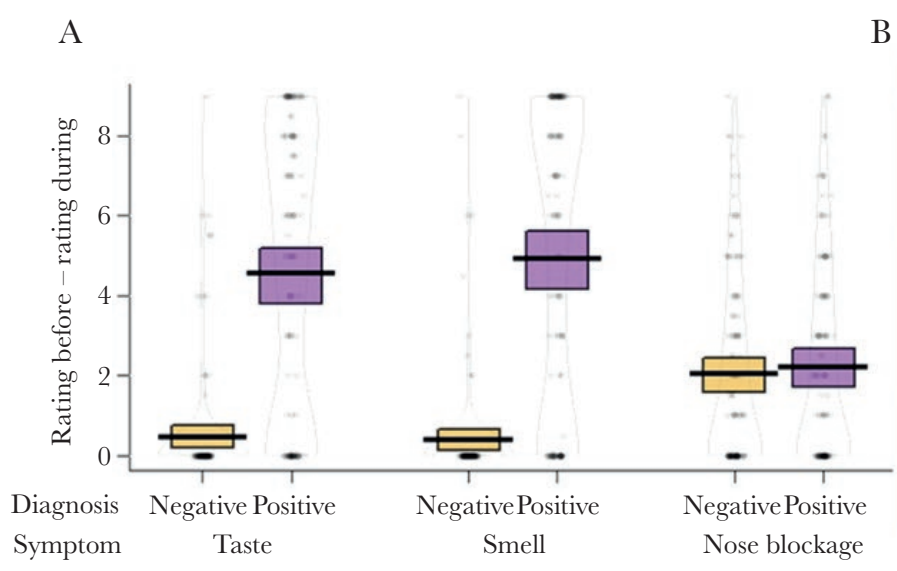

\begin{tabular}{|c|c|c|c|}
\hline & Negative & Positive & $P$-value \\
\hline Taste change total (n) & $\begin{array}{c}0.5 \pm 1.5 \\
(112)\end{array}$ & $\begin{array}{l}4.5 \pm 3.6 \\
(112)\end{array}$ & $<.0001$ \\
\hline $\begin{array}{l}\text { Those who reported } \\
\text { taste change (n) }\end{array}$ & $\begin{array}{l}2.1 \pm 2.3 \\
(20)\end{array}$ & $\begin{array}{c}6.3 \pm 2.6 \\
(81)\end{array}$ & $<.0001$ \\
\hline Smell change total (n) & $\begin{array}{c}0.4 \pm 1.5 \\
(112)\end{array}$ & $\begin{array}{l}4.9 \pm 3.8 \\
(112)\end{array}$ & $<.0001$ \\
\hline $\begin{array}{l}\text { Those who reported } \\
\text { smell change (n) }\end{array}$ & $\begin{array}{c}4.8 \pm 2.6 \\
(9)\end{array}$ & $\begin{array}{c}7.1 \pm 2.4 \\
(76)\end{array}$ & .03 \\
\hline $\begin{array}{l}\text { Nose blockage } \\
\text { change total (n) }\end{array}$ & $\begin{array}{c}2.1 \pm 2.6 \\
(112)\end{array}$ & $\begin{array}{c}2.2 \pm 2.5 \\
(112)\end{array}$ & .7 \\
\hline $\begin{array}{l}\text { Those who reported } \\
\text { smell change (n) }\end{array}$ & $\begin{array}{c}3.2 \pm 3.5 \\
(9)\end{array}$ & $\begin{array}{c}2.4 \pm 2.6 \\
(76)\end{array}$ & .5 \\
\hline
\end{tabular}

Figure 3. Degree of taste, smell, and nose blockage in COVID-19 positives and negatives. A, Histogram showing the change in rating during the illness minus the rating before the illness for taste, smell, and nose blockage. No change is coded 0, and the highest change is 9. COVID-19 positives are represented in purple, and COVID-19 negatives are represented in orange. B, Table of mean \pm SD for COVID-19 positives and negatives in general and for those reporting changes of taste or smell. Scores for taste, smell, and nose blockage were evaluated on a 1-10 scale. Pvalues for the difference in the magnitude of change between COVID-19 positives and negatives were calculated using a 2-sided $t$ test. Abbreviation: COVID-19, coronavirus disease 2019.

AUC of 0.78 (Classifier 16) (Supplementary Table 1), as compared with an AUC of 0.81 using quantitative smell descriptor.

The rate of positive tests out of total PCR tests at the time of participant recruitment was calculated to be $8 \%$ based on data from the Israeli Ministry of Health. By incorporating this factor (see the "Methods" section), a PPV of 0.68 and an NPV of 0.97 for both classifiers were obtained. A probability calculator based on Classifiers 2, 3, and 14 (Supplementary Data) is available in GitHub (https://github.com/KimAsseo/Hadassah_COVID-19).

\section{DISCUSSION}

We have established the prevalence and degree of decrease in taste and smell in patients who were eligible to receive PCR swab tests during the COVID-19 pandemic and found significant differences in PCR-positive and PCR-negative patients.

The change in ability to smell is not related to nasal obstruction, as nose blockage was low, as previously demonstrated [6, 15]. Taste and chemesthesis changes strongly correlate with smell change (in agreement with [6]). Taste changes are more

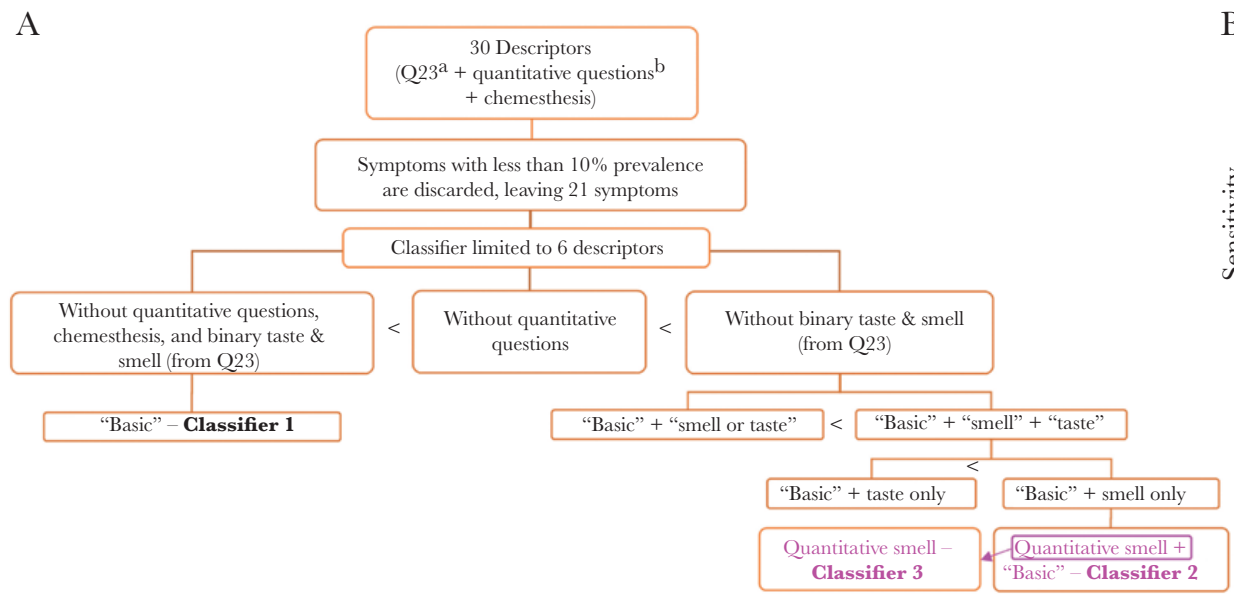

*Includes all symptoms in question 23 excluding "no symptoms", coding "other" to "fatigue" and "dizziness", and grouping eye symptoms

**Includes taste, smell and nose blockage.

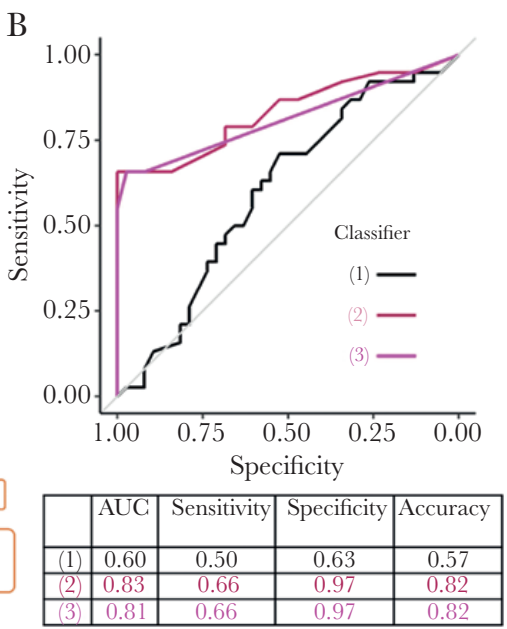

Figure 4. Descriptor selection process for classifiers. A, The flowchart depicts the total symptoms selected for the classifiers. Upon limiting the number of descriptors for the classifiers and excluding chemosensory symptoms as described, Classifier 1 was created. Different combinations of symptoms established better classifiers than Classifier 1 , with those using quantitative questions exhibiting better performance than those using binary ones. The classifier using "smell" and "taste" as separate descriptors, rather than "smell or taste" as a single joint descriptor, showed better performance. The "basic" + smell only descriptor outperformed the "basic" + taste only descriptor, resulting in Classifier 2. Finally, the smell only descriptor was tested alone without all other "Basic" symptoms, resulting in Classifier 3. B, The graph illustrates ROC curves for the classifiers considered, and the table shows the statistical parameters for the ROC curves. For the quantitative descriptors, the coefficients correspond to a descriptor scaled to the 0-1 range. "Includes all symptoms in question 23, excluding "no symptoms," coding "other" to "fatigue" and "dizziness," and grouping eye symptoms. "Includes taste, smell, and nose blockage. Abbreviations: AUC, area under the curve; ROC, receiver operating characteristics. 
common than smell changes in negatives, and chemesthesis changes are less common than taste and smell changes in positives, leading to odds ratios for these chemosensory modalities that are high, but lower than for olfaction.

All taste modalities in COVID-19 patients were impacted together (or not at all). This is of interest for understanding the pathophysiology of the disease: A recent study suggests $\mathrm{CoV}-2$ infection of non-neuronal cell types expressing ACE2 and TMPRSS2 as the mechanism underlying COVID-19-related anosmia [16], but the reason for COVID-19 ageusia is less clear $[11,17]$. Our results support the idea of impairment of supporting cells or tissues, rather than of Type 2 taste receptor cells, which express bitter, sweet, and umami taste receptors, or Type 3 taste receptor cells, which express sour-sensing channels [18]. Routine addition of taste questions for patient screening is not warranted, as these questions did not contribute to classifier performance. Nevertheless, patients with prior conditions of impaired olfaction (estimated 5\% of population [19]) require a suited classifier.

We present 3 versions of classifiers: The first is based on 4 yes/ no questions (muscle ache, lack of appetite, fever, sore throat) and quantitative smell change. The second version uses only quantitative smell change and has a similar AUC. The third (suited for participants with preexisting olfactory impairment) is based on 5 yes/no questions (muscle ache, lack of appetite, fever, sore throat, breath difficulty) and quantitative taste change. The probabilities for COVID-19 based on these calculators are available via GitHub.

Our best-performing classifier (Classifier 2; AUC, 0.83) used quantitative smell change, muscle ache, lack of appetite, fever, and (negative contributing) sore throat. Performed in parallel to Gerkin et al. [10], our study similarly included both binary and quantitative questions regarding taste and smell as 2 separate indicators. Our results for positive patients are in overall agreement with Parma et al. [6] and Gerkin et al. [10]. This is striking in view of the different methods employed for recruitment (targeting smell and change impairment worldwide vs PCR-positive COVID-19 patients in Israel), data collection (online survey vs telephone interviews), and quantitative scales (100-point visual analog scale [VAS] vs 1-10 scale in this study). In essence, both Gerkin et al. and the current study suggest that quantitative smell change is the best predictor of COVID-19 in single- and cumulative-feature models and is better than binary features. The superior performance of the classifier (AUC of $0.83 \mathrm{vs}$ 0.72 in [10]) is probably due to a more realistic representation of our sample, in which chemosensory losses were not overrepresented.

With the increasing public awareness of smell impairment as COVID-19 characteristic symptoms, individuals presenting smell and taste changes are now more likely to be suspected of having COVID-19. By considering other symptoms and the severity of chemosensory change, our calculators provide a free, fast, and easy-to-use tool that can provide immediate answers for patients awaiting their PCR swab test results and potentially decrease anxiety in negative patients who experience mild smell and taste impairments.

\section{Study Limitations}

The method of patient recruitment is one of the limitations of this study: Social media-based recruitment may limit participants' representation, as this method targets mostly younger patients with internet access and social media accounts. Word of mouth recruitment was used as well, and it also contributes to creating a sample that is not necessarily representative of the general population. Male and female patients were not fully matched across positives (64\% males) and negatives (48\% males), in accordance with the higher percentage of males (56\%) among COVID-19 patients in Israel [20].

False-negative results of the RT-PCR test have been reported to occur in $\sim 30 \%$ of COVID-19 patients [21]. At 3 -week follow-up, none of the negative patients who were not recovered at the time of their initial questionnaire $(\sim 50)$ had had a repeat RT-PCR test, so false negatives could not be ruled out. Serology tests were performed for a sample of 5 negative patients who reported taste and/or smell impairments. All 5 had negative serology using the LIAISON SARS-CoV-2 S1/S2 IgG assay [22], confirming that these patients are likely true negatives.

Importantly, our classifiers are not SNOUT ("Sensitive test when Negative rules OUT the disease") but can definitely be referred to as SPIN ("Specific test when Positive rules IN the disease").

Our sample was composed of slightly to moderately ill patients. It should also be kept in mind that our data are specific to Israeli patients and reflect to some degree the criteria for PCR test eligibility during recruitment (fever and dry cough were sufficient for PCR testing, but change of smell and/or taste alone was not).

\section{CONCLUSIONS}

The resurging pandemic puts the clinic and public health authorities in a scenario that is not usual for modern medicinenamely, limited resources require, or may require in the future, prioritization of testing and treatment. The fact that our sample contained PCR-positive and PCR-negative ambulatory patients, all suspected to have COVID-19 before PCR testing, enabled the development of symptom-based classifiers.

Our results suggest that ranking of the ability to smell before and during illness is an excellent practical approach to identify COVID-19-positive patients that offers reasonably high predictive capability (specificity, 97\%; accuracy, $82 \%$ ). An additional classifier is available for patients with prior olfactory impairments (Supplementary Figure 1). 
Based on the classifiers developed in this work, we propose a simple calculator that can be used to prioritize testing (available at https://github.com/KimAsseo/Hadassah_COVID-19). Additionally, a high-performance classifier may potentially capture false-negative PCR test results of high-scoring individuals. The current study provides a practical tool for assessing potential COVID-19 patients.

\section{Supplementary Data}

Supplementary materials are available at Open Forum Infectious Diseases online. Consisting of data provided by the authors to benefit the reader, the posted materials are not copyedited and are the sole responsibility of the authors, so questions or comments should be addressed to the corresponding author.

\section{Acknowledgments}

We thank the Global Consortium for Chemosensory Research (GCCR) team for inspiring the questionnaire used in this research. We thank Yehuda Tarnovsky for help with patient recruitment. The graphical abstract was created with BioRender.com.

Financial support. M.Y.N. is supported by Israel Science Foundation grant \#1129/19. This work was supported in part by the Edmond de Rothschild Foundation. H.K. is a recipient of the Uri Zehavi Scholarship.

Potential conflicts of interest. All authors: no reported conflicts of interest. All authors have submitted the ICMJE Form for Disclosure of Potential Conflicts of Interest. Conflicts that the editors consider relevant to the content of the manuscript have been disclosed.

Author contributions. N.K. and M.Y.N. initiated the research, N.K., H.K., and M.N. recruited the patients, N.K., M.Y.N., Y.B., and M.M. designed the research, S.I., M.M., R.N.P., and M.Y.N. supervised the research, M.N assisted in the wording and translation of the questionnaires, H.K. carried out the interviews, H.K. and M.Y.N. drafted the manuscript, and K.A. and Y.B. performed the statistical analysis and created the figures and tables. N.K.'s participation in this study was performed in fulfillment of the requirements toward their MD degree at the Hebrew University of Jerusalem. All authors contributed to the writing and approved the final manuscript.

Ethics approval. The study was conducted in accordance with the Helsinki Declaration, and the required ethical approval was granted (reference number HMO-0236-20).

Patient consent. Written informed consents for publication of patients' clinical details were obtained from the patients.

\section{References}

1. Zhu N, Zhang D, Wang W, et al; China Novel Coronavirus Investigating and Research Team. A novel coronavirus from patients with pneumonia in China, 2019. N Engl J Med 2020; 382:727-33.
2. Machado C, Gutierrez JV. Anosmia and ageusia as initial or unique symptoms after SARS-COV-2 virus infection. Preprints 2020040272 [Preprint]. 16 April 2020. Available at: https://www.preprints.org/manuscript/202004.0272/v1. Accessed 26 July 2020.

3. Levinson R, Elbaz M, Ben-Ami R, et al. Time course of anosmia and dysgeusia in patients with mild SARS-CoV-2 infection. Infect Dis (Lond) 2020; 52:600-2.

4. Moein ST, Hashemian SM, Mansourafshar B, et al. Smell dysfunction: a biomarker for COVID-19. Int Forum Allergy Rhinol 2020; 10:944-50.

5. Gilani S, Roditi R, Naraghi M. COVID-19 and anosmia in Tehran, Iran. Med Hypotheses 2020; 141:109757.

6. Parma V, Ohla K, Veldhuizen MG, et al. More than smell-COVID-19 is associated with severe impairment of smell, taste, and chemesthesis. Chem Senses 2020; 45:609-22.

7. Whitcroft KL, Hummel T. Olfactory dysfunction in COVID-19: diagnosis and management. JAMA 2020; 323:2512-4.

8. Menni C, Valdes AM, Freidin MB, et al. Real-time tracking of self-reported symptoms to predict potential COVID-19. Nat Med 2020; 26:1037-40.

9. Menni C, Sudre CH, Steves CJ, Ourselin S, Spectora TD. Quantifying additional COVID-19 symptoms will save lives. Lancet 2020; 395:e107-8.

10. Gerkin RC, Ohla K, Veldhuizen MG, et al. Recent smell loss is the best predictor of COVID-19 among individuals with recent respiratory symptoms [published online ahead of print December 25, 2020]. Chem Senses 2020; doi:10.1093/ chemse/bjaa081

11. Chaudhari N, Roper SD. The cell biology of taste. J Cell Biol 2010; 190:285-96.

12. Robin X, Turck N, Hainard A, et al. pROC: an open-source package for R and S+ to analyze and compare ROC curves. BMC Bioinformatics 2011; 12:77.

13. Bagheri SH, Asghari A, Freidin M, Shamshiri AR, Kabir A, Kamrava SK, Jalessi M, Mohebbi A, Alizadeh R, Honarmand AA, Ghalehbaghi B, Salimi A. Coincidence of COVID-19 epidemic and olfactory dysfunction outbreak. Med J Islam Repub Iran 2020; 34:62.

14. Centers for Disease Control and Prevention. Symptoms of coronavirus. Available at: https://www.cdc.gov/coronavirus/2019-ncov/symptoms-testing/symptoms.html Accessed 24 July 2020.

15. Salmon Ceron D, Bartier S, Hautefort C, et al. Self-reported loss of smell without nasal obstruction to identify COVID-19. The multicenter Coranosmia cohort study. J Infect 2020; 81:614-20.

16. Brann DH, Tsukahara T, Weinreb C, et al. Non-neuronal expression of SARSCoV-2 entry genes in the olfactory system suggests mechanisms underlying COVID-19-associated anosmia. Sci Adv 2020; 6:eabc5801.

17. Cooper KW, Brann DH, Farruggia MC, et al. COVID-19 and the chemical senses: supporting players take center stage. Neuron 2020; 107:219-33.

18. Roper SD, Chaudhari N. Taste buds: cells, signals and synapses. Nat Rev Neurosci 2017; 18:485-97.

19. Hummel T, Landis BN, Hüttenbrink KB. Smell and taste disorders. GMS Curr Top Otorhinolaryngol Head Neck Surg 2011; 10:Doc04.

20. Ministry of Health of Israel. Corona lab tests in Israel. Available at: https://data gov.il/dataset/covid-19/resource/dcf999c1-d394-4b57-a5e0-9d014a62e046/ download/corona_lab_tests_ver_0027.csv. Accessed 2 August 2020.

21. Weissleder R, Lee H, Ko J, Pittet MJ. COVID-19 diagnostics in context. Sci Trans Med 2020; 12:eabc1931.

22. DiaSorin. LIAISON SARS-CoV-2 S1/S2 IgG: the fully automated serology test for the detection of SARS-CoV-2 IgG antibodies. Available at: https://www. diasorin.com/sites/default/files/allegati/liaisonr_sars-cov-2_s1s2_igg_brochure.pdf.pdf. Accessed 2 June 2020. 